\title{
POLYURIA (DIABETES INSIPIDUS) IN BASAL CEREBRAL DISEASE.
}

\author{
BY HENRY HANDFORD, M.D., M.R.C.P.LOND. \\ Physician to the General Hospital, Nottingham.
}

THE following two cases are the only examples of this phenomenon with which I have met in a somewhat extensive hospital experience. And the second, in which the fact rests only upon the statements made by the patient and her friends, is sufficiently indefinite to be cast aside, were it not so amply supported by the details of the first case.

Gowers says, "The urine is seldom altered in composition by organic brain disease, although polyuria rarely, and glycosuria occasionally, have been produced by disease of the pons and medulla oblongata."

The analogy with diabetes from puncture of the floor of the fourth ventricle seems to be very close. It would appear probable that the pressure of a gumma paralysed the vasomotor centre for the kidney in the medulla, the absence of polyuria until the latter part of the second attack, and its continuance when all other symptoms were improving, is difficult to explain. But we must accept as a fact that a tumour may grow all round a nerve without pressing upon it sufficiently to interfere with its function, and that of several nerves to all outward appearance equally involved and pressed upon by a tumour some may be completely paralysed, and others quite uninjured. The direct and indirect pressure upon the brain substance is also open to many sources of variation. Hence it is useless to speculate upon the escape of the renal centre until a particular time, and the absence, or slight degree of, improvement when the other symptoms of pressure upon the nerve trunks and nerve tissues were so greatly diminishing. 
The frequency with which iodide of potassium and mercury are given for diseases of all kinds without any such diuresis following, renders it clear that the treatment was not in any way responsible for the phenomenon.

I have detailed the first case very fully in order that it may bear internal evidence of the accuracy of the diagnosis, both as to the presence of a tumour and as to its syphilitic nature.

Case 1.-Julia M., aged 27, lacewinder, admitted into the General Hospital, Nottingham, Oct. 15, 1891, complaining of fits, \&c.

Her father died in the hospital of chronic Bright's disease. Her mother died of dropsy: brothers and sisters are healthy. Patient had fits as a child, and five years ago fell and struck her head, remaining unconscious for two or three hours. She completely recovered. Before marriage she was for some years a prostitute; had venereal disease, rashes, and sore throat. She has been married $6 \frac{1}{2}$ years, and has two children, the youngest eight months old.

Her present illness commenced about eight months ago, three weeks after her last confinement. She noticed that her left eye was getting "crooked," and she frequently vomited. (The vomiting continued till six weeks ago).

She next found that her left arm and leg were getting weak. She could not hold the baby on her arm.

She suffered from double vision, headache, and a feeling of pain, and "pins and needles" shooting down the left side. This peculiar numb feeling usually only continues about five minutes, but when it lasts a quarter of an hour or longer she loses consciousness. She can tell when these "fits" are coming on.

On admission she was found to be well nourished and fairly intelligent, but her memory was evidently failing.

The grasp of the left hand is markedly weaker than the right: -dynamometer, L. 35, R. 65. The left leg is also evidently weaker than the right. There is no facial paralysis, and the tongue is protruded straight. Sensibility to touch and to pain are normal, except perhaps over the left arm, where they are both somewhat deficient. The muscular sense is impaired on the left side. Sensation to heat and cold normal. Taste impaired, but not lost.

The plantar reflexes are exaggerated, especially on the left side; the patella reflexes also; no ankle clonus. The left wrist, triceps and biceps jerks are exaggerated. 
There is complete paralysis of the right external rectus; and marked weakness of the left external and internal recti. There is nystagmus on the left side, and some proptosis. No optic neuritis, but masses of pigment showing remains of old choroiditis. Left eye myopic, right hypermetrophic.

Ten days after admission the note runs:-Since admission she has had 3 to 9 fits daily, never losing consciousness. She has vomited once or twice for the past two days. The headache has been more severe, and is general in distribution. It is better at night and allows her to sleep. She is up in the afternoon and can get about.

Sensory Aura.-She always knows when the fits are coming on by a pain in the left temple, followed by a feeling of " pins and needles " in the left arm, commencing in the fingers and passing up the arm. This is from five to ten minutes before the fit commences. After the "pins and needles" in the arm comes a pain in the left flank just below the tenth rib, passing down the outer side of the thigh and leg to the toes which are then flexed, and immediately the leg becomes rigid and remains extended. She then rubs the left foot with the right. In the meantime the fingers of the left hand are flexed, and the arm becomes extended until she grasps the wrist with the right hand and draws it across the chest. She does not utter any cry. The fit lasts about two minutes. The mouth is drawn a little to the left. The procedure is exactly repeated every time. She becomes drowsy after the fits, and complains of the headache becoming much more severe and general.

Nov. 2.-She has been taking iodide of potassium, gr. $\mathbf{x}$., three times a day, and pil. hydrargyri gr. $3 \frac{3}{4}$, twice a day, ever since Oct. 20. She is now gaining power in the left arm and leg, and the fits are not nearly so frequent. The aura has ceased, so that now the fits come on without warning. And instead of being preceded by tingling in the leg, they are followed by pain in the left middle finger going up to the elbow. The pain in the head has been much less severe.

Dec. 2.-For the past few weeks she has never had more than one slight fit daily and often none for several days.

Now the fits commence in the leg with rigidity of the muscles; the tingling and sensory phenomena have ceased.

Jan. 8.- She has been away to the convalescent hospital for a fortnight, and during that time has only had one fit.

The left leg seems now as strong as the right, and she can walk several miles: There is no stiffness, but the left knee-jerk is greatly increased. 
She has had no headache for several weeks. The reactions of the pupils are normal, but the left is slightly larger than the right. There is still weakness of both external recti and nystagmus on abduction. No change in the fundus besides the remains of old choroiditis. She is now an out-patient.

Jan. 21.-Since she left the hospital a perforation of the soft palate has formed close to the uvula, which seems likely to slough off. There is also a syphilitic pharyngitis.

March 7.-Patient was readmitted. She had not attended since Jan. 21.

She has had much headache and romiting; and is now very feeble and emaciated. She is hardly able to stand, and usually lies in a semi-comatose state. The left knee jerk is exaggerated, the right almost absent. There is no distinct clonus on either side, but some slight rigidity of both legs. Slight impairment of common sensation over the right hip and flank. The condition of the palate and fauces is unaltered since Jan. 21st. She can move the tongue freely: it is dry and coated with a brown fur. Temperature 97.2.

There is complete paralysis of the left external rectus, and nearly complete of the right, Pupils more than half dilated. Left disc swollen: vessels curving over the edge. Right disc slightly hazy. Left globe prominent.

April 4.-Patient has been treated by iodide of potassium, gr. $x$, three times a day, together with mercurial inunction.

For a few days after re-admission she was delirious, especially at night; and for a week or two she lay in a semi-conscious, drowsy, state; for the first fortnight the urine was scanty.

From March 15 the quantity of urine almost suddenly began to increase enormously, ranging from $100 \mathrm{oz}$. to $130 \mathrm{oz}$. in addition to what was lost with the motions. The sp. gr. varied between 1,006 and 1,008; and the urine was quite free from albumin and sugar.

From April 12 to May 2 patient again went to the convalescent hospital and improved in general condition, but the urine still continued between 100 and 140 ounces daily, besides what was necessarily lost.

On May 25 the iodide of potassium was stopped, and zi. of liq. hydrargyri perchloridi alone given. The quantity of urine was not materially affected, although it continued to be carefully measured for ten days further. She was then made an outpatient.

A month later, June 22, she was again re-admitted for two days, and the polyuria still continued. The paralysis of the 
external recti, the perforation of the soft palate, and the exaggerated knee jerk remained. Otherwise she seemed well. She was advised to continue medicine once a day for another six months; but she did not return, and has been lost sight of.

The second case is not nearly so definite. She was admitted in January, 1890, under Dr. Ransom, to whom I am indebted for permission to use the case.

The patient was a woman, aged 34 , who had had two children and three miscarriages, with a very definite history of syphilis.

During the past year she has suffered much from headache. In April, 1889, i.e., nine months ago, she began to suffer from intense thirst. In one afternoon she drank two dozen half-pint bottles of soda water. She did not drink intoxicating liquors. In one day she has passed as much as three gallons and half a pint of urine.

This polyuria ceased two months ago, when the headache became more severe, and drooping of the left eye-lid began. She has had no vomiting.

She is slow in answering questions, and seems to forget words. There is no paralysis of face, arms, or legs ; but there is complete ptosis of the left eye-lid, and the left pupil is dilated and does not re-act to light or accommodation. No optic neuritis. Sensation is unaltered. She has had several slight epileptic seizures.

She was treated with pot. iod. gr. x., three times a day, and improved considerably. The polyuria did not return while she was in the hospital. A gumma was diagnosed in the temporosphenoidal lobe, involving the third nerve as it emerges from the base of the brain. 